\title{
Macht und Ohnmacht des Geldes im Privaten - Zur Dynamik von Individualisierung in Paarbeziehungen
}

\author{
Von Werner Schneider, Andreas Hirseland, Wolfgang Ludwig-Mayerhofer und \\ Jutta Allmendinger
}

Zusammenfassung: Mit fortschreitender Modernisierung werden nicht nur die kategoriale Ordnung der >ersten Moderne < und damit deren soziale und kulturelle Leitsemantiken brüchig, ambivalent, hybride. Vielmehr erfolgt ein grundlegender Wandel moderner Basisinstitutionen und Basisprinzipien des Sozialen/Gesellschaftlichen. Vor dem Hintergrund dieser Grundannahmen der Theorie reflexiver Moderne diskutiert der Beitrag die Frage nach den Machtwirkungen, Ungleichheits- und Individualisierungseffekten von Geld in modernen Doppelverdiener-Paarbeziehungen. Die dort anzutreffenden Geldpraktiken verweisen in ihren spezifischen Zurechnungen von >MeinDein-Unser $<$ auf eine zunehmende Reflexivität der intimen Vergemeinschaftung von Männern und Frauen, in welcher das einfach-moderne >Entweder-Oder $<$ von Geld versus Liebe durch typische paarinterne Konstruktionen von >Beziehungsgeld $<$ in ein >Sowohl-als-auch $<$ transformiert wird. Darin zeigen sich Ansätze einer Neuordnung des Verhältnisses von Privatheit/Öffentlichkeit als (geschlechts-)spezifischer Macht- und Herrschaftsfiguration der ersten Moderne hin zu einer privatisierten, in die Individuen hinein verlagerten >Selbst-Herrschaft $<$, die beide Geschlechter ergreift, jedoch mit unterschiedlichen ungleichheitsrelevanten Voraussetzungen und Folgen. Damit zielt der Beitrag auf eine empirisch fundierte, >reflexiv moderne < und darin insbesondere ungleichheits-/ macht- und herrschaftssensible Soziologie des Geldes im Privaten.

\section{Geld in Paarbeziehungen - ein Individualisierungsmotor?}

Geld und Liebe wurden in der Soziologie bislang eher selten und zumeist getrennt voneinander verhandelt. Sind schon - auf welchen theoretischen Prämissen und empirischen Fundamenten auch immer beruhende - Analysen jeweils zu Geld (z.B. Deutschmann 1999, 2002; Paul 2004) oder zu Liebe (z.B. Hahn/Burkart 1998, 2000) eher rar, so fand das Verhältnis beider historisch-empirisch wie theoretisch erst recht wenig Beachtung. ${ }^{1}$ Auf der einen Seite richtete sich der nach sozialen Ungleichheiten forschende Blick bei Einkommen und Vermögen in der Regel entweder auf Individuen (etwa: deren Arbeitslohn) oder aber als Haushaltseinkommen auf Haushalte. Lange Zeit ausgeblendet blieb, wie Einkommen, Vermögen oder auch Schulden in der Familie bzw. in der Paarbeziehung be- und verhandelt, diskutiert und distribuiert werden. Auf der anderen Seite rückt bis heute das Thema >Geld< umso weiter in den Hintergrund, verschwindet gleichsam hinter dem Schleier der Liebe, je mehr Paarbeziehungen und Familien unter dem Blickwinkel von personal-affektiver Vergemeinschaftung betrachtet werden (Allmendinger et al. 2001). ${ }^{2}$

1) Vgl. Dackweiler/Hornung (2003), Illouz (2003), Wimbauer (2003) (siehe hierzu auch Nassehi 2005); verschiedene Ansätze einer theoretischen Fassung des Verhältnisses von >Geld und Liebe< im bürgerlichen Familienhaushalt, wie z.B. in familienökonomischen Ansätzen (im Anschluss an Becker 1981), in einer kritisch-theoretischen bzw. marxistischen Auseinandersetzung mit der Familienideologie des Kapitalismus (z.B. Millhofer 1973) oder im feministischen Diskurs (Delphy/Leonard 1992; Walby 1990), bleiben hier aus Platzgründen undiskutiert.

2) Ein Blick in neuere einschlägige Werke zu Ehe und Familie bzw. insbesondere zur Soziologie der Zweierbeziehung macht dies deutlich (vgl. z.B. Lenz 2003; Herrmann 2001; Hoffmeister 2001; Hohenester 2000): Hauptthemen sind hier Beziehungsphasen und Beziehungsarbeit, Identität, Emotionen, >Liebe . Für einen Überblick zum Forschungs- und Diskussionsstand zum Thema >Geld in Familien/Paarbeziehungen $<$ vgl. Schneider/Wimbauer (2004); Ludwig-Mayerhofer (2004). 
Diese soziologische Beobachtungsanordnung und ihre begrifflich-kategoriale Logik werden jedoch nicht zuletzt im Zuge der Modernisierungsschübe der zweiten Hälfte des 20. Jahrhunderts durch empirische Entwicklungen, vor allem die steigende Erwerbsbeteiligung von Frauen und das durch eigenwertige Berufstätigkeit von ihnen >selbst verdiente< Einkommen, grundlegend in Frage gestellt (Rubery/Smith/Fagan 1999, Blossfeld/Drobnič 2001). Das >eigene Geld< scheint - so eine prominente und weit verbreitete Annahme - als ein wichtiger, ja als der Motor von Individualisierung im Privaten zu fungieren, indem es Frauen ein >eigenes Leben< jenseits der für die >erste, einfache Moderne< (Beck/Lau in diesem Band) charakteristischen, kulturell festgeschriebenen Eindeutigkeit der geschlechtsspezifischen Aufgabenverteilung in Ehe und Familie ermöglicht (z.B. Beck-Gernsheim 1983, 1998). In dieser nachholenden Modernisierung mit ihrer geldvermittelten Freisetzung von Frauen aus dem institutionellen Regime naturalisierter Geschlechtertypisierungen sind nun die (männlichen wie weiblichen) Individuen zu permanenten Aushandlungen und kontinuierlicher Konstruktion und Rekonstruktion ihres gemeinsamen Beziehungsalltags genötigt. Als mögliche Folge hiervon dynamisieren, öffnen und pluralisieren sich die intimen Beziehungswelten von Männern und Frauen, relativieren sich Macht- und Abhängigkeitsverhältnisse zwischen ihnen, vermindern sich gar Ungleichheiten (z.B. Giddens 1992). Diesen Annahmen gemäß bewirkt die >gesellschaftliche Macht des Geldes` (Deutschmann 2002) die individualisierte, reflexive Um- und Neuordnung von Paarbeziehungen, die bis in die zweite Hälfte des 20. Jahrhunderts hinein vom Prozess der Modernisierung nur im institutionellen Rahmen des bürgerlichen Ehe- und Familienmodells als >Basisinstitution< einer in Bezug auf die Geschlechterverhältnisse >halbierten Moderne< erfasst wurden (Beck 1986; Beck/Lau in diesem Band).

So betrachtet erweist sich die weitgehende soziologische Vernachlässigung des Themas >Geld in intimen Paarbeziehungen als Spiegelbild und Nachhall jener >einfach-modernen< gesellschaftlichen Selbstbeschreibung, die entlang eindeutiger begrifflicher Unterscheidungen und Dichotomien wie männlich oder weiblich, geldvermitteltes Arbeitsleben oder um Intimität zentriertes Privatleben, Rationalität oder Emotionalität operiert. Die Theorie reflexiver Modernisierung hingegen geht davon aus, dass mit der fortschreitenden Moderne in ihrer radikalisierten Umsetzung moderner Basisprinzipien ein grundlegender Wandel einfachmoderner Basisinstitutionen erfolgt und mithin die kategoriale Ordnung der ersten Moderne und ihre Leitsemantiken brüchig, ambivalent, hybride werden (Beck/Lau 2004). Daraus folgt nicht zuletzt für die sozialwissenschaftliche Beobachterperspektive, sich bei der Analyse dieses Wandels keinesfalls unhinterfragt jener überkommenen Begriffe, Kategorien und Semantiken zu bedienen, sondern im Sinne eines >methodologischen Kosmopolitismus< diese selbst $\mathrm{zu}>$ de-konstruieren< sowie neue Konzepte und Kategorien empirisch begründet zu >re-konstruieren $<($ Beck/Lau in diesem Band).

Insofern verfolgt der vorliegende Beitrag ein zweifaches Ziel: Zum einen geht es um die empirisch zu klärende Frage nach den aktuellen Transformationsprozessen in der institutionellen Ordnung von >Geld und Liebe < bzw. genauer: um den behaupteten, mit dem >eigenen Geld von Frauen einhergehenden Wandel geschlechtsspezifischer Macht-, Herrschafts- und Ungleichheitsverhältnisse im Privaten. Zum anderen ist zu klären, auf welchen begrifflichtheoretischen Fundamenten die empirische Analyse des behaupteten Zusammenhangs von Geld und Individualisierung in privaten Beziehungswelten überhaupt stehen kann. Unsere empirisch gewonnene Kernthese hierzu lautet, dass sich mit dem >eigenen Geld< beider Partner die Bedingungen für die Produktion und Reproduktion von Geschlechterungleichheit in der intimen Paarbeziehung grundlegend verändern. Allerdings werden wir zeigen, dass dies nicht zwingend in der häufig unterstellten Art und Weise einer radikalisiert ins Private reichenden, aus der öffentlichen Sphäre der Geldwirtschaft sich speisenden >Individualisierungs-Macht< des Geldes erfolgt. Denn zu klären ist, was als >Geld < in intimen Paarbezie- 
hungen gilt (bzw. wie geltend gemacht wird), welche Bedeutungen ihm zukommen und welche entsprechenden Macht- bzw. Ungleichheitswirkungen es entfaltet.

Hierzu gehen wir in drei Schritten vor: Der erste Schritt (Kap. 2) versucht, die >Basisselbstverständlichkeiten< (Beck/Lau in diesem Band) der ersten Moderne zum Verhältnis von Geld und Liebe im bürgerlichen Ehe- und Familienmodell freizulegen und dadurch zu zeigen, wie die behauptete Modernisierung der intimen Paarbeziehung durch die individualisierende Wirkung >eigenen Geldes< im Privaten begrifflich-konzeptionell selbst noch in den Beschreibungskategorien dieser ersten Moderne verfangen bleibt. Der zweite Schritt (Kap. 3) skizziert dem entgegen begrifflich-konzeptionelle Bausteine einer empirisch begründeten >Soziologie des Geldes in intimen Paarbeziehungen<, deren empirische Befunde und modernisierungstheoretische Konsequenzen zum Zusammenhang von Geld, Individualisierung und Macht, Herrschaft, Ungleichheit in Paarbeziehungen abschließend diskutiert werden (Kap. 4).

\section{Zur institutionellen Ordnung von Geld und Liebe in der Moderne}

\subsection{Das bürgerliche Ehe-/Familienmodell und die Zähmung des Geldes im Privaten}

Mit der Entstehung der modernen Arbeitswelt geht die Ausdifferenzierung eines als >besondert< gedachten lebensweltlichen Raumes einher: die private Welt der bürgerlichen Kleinfamilie. Diesem Ehe- und Familienmodell zufolge hat sich die Partnerwahl nicht mehr, wie in der traditionalen Gesellschaft, nach ökonomischen Gesichtspunkten und kollektiven Verwandtschaftsinteressen zu richten, sondern wird durch >romantische Liebe (vgl. z.B. Tyrell 1987; Luhmann 1982) gesteuert. Das >symbolisch generalisierte Kommunikationsmedium Liebe < (Parsons 1951; Luhmann 1982, 1984) mit seinem Verhaltensmodell der >Passion $<$ markiert für die bürgerliche Familie eine nach außen abgeschottete, gleichwohl gesellschaftlich kontrollierte Privatsphäre, in der die (Ehe-)Partnerbeziehung die zentrale Achse dieser neuen, intimisierten Beziehungswelt bildet. Die handlungsleitenden Normen für die Intimbeziehung der Lebenspartner unterliegen nicht nur den Maximen wechselseitigen Vertrauens, sondern auch und vor allem der beiderseitigen personalen Offenbarung: Es gibt keine >Bagatellen in der Liebe<, alles Erlebte ist mitteilenswert, alles findet in dieser Beziehung kommunikative Resonanz (Luhmann 1982). So werden aufgrund der Gesamtheit ihrer Persönlichkeit die Lebenspartner zum jeweils >signifikanten Anderen< (Mead 1973) füreinander, erschaffen in der alltäglichen intimen Binnenkommunikation ihre je besondere gemeinsame Beziehungswelt und stellen sie in diesem fortwährenden >Gespräch < auf Dauer (Berger/Kellner 1965). Diese am Erleben von alter ego und dessen jeweiliger Höchstrelevanz orientierte Form wechselseitiger Bezogenheit soll über symbolische Tauschtabus stabilisiert werden, nach denen letztlich $>$ Liebe nur gegen >Liebe getauscht werden soll, kann, darf.

In der Idealisierung des >trauten Heims « wird das bürgerliche Ehe- und Familienmodell symbolisch aufgeladen und konnotiert >liebevolle< emotionale Nähe, Sicherheit, Ruhe, Harmonie. Es verheißt Erholung, Regeneration/Reproduktion und gilt als Refugium und Bollwerk gegen das entfremdende gesellschaftliche Außen, gekennzeichnet durch Anonymität, rationale Distanz, Effizienz und Konkurrenz, (Maihofer/Böhnisch/Wolf 2001; Segalen 1998). So gerinnen Ehe und Familie dem modernen Individuum zum bevorzugten Ort der Einlösung des Wunsches nach emotionaler, personalisierter Vergemeinschaftung und nichtpartikularer, umfassender Selbst-Erfahrung. Sie bilden damit den modernespezifischen Kontrast zu den abstrakten - d.h. von konkreten Individuen abstrahierenden - Vergesellschaftungsprozessen durch zunehmend markt- und damit geldvermittelte (Re-)Produktion und Konsumption in den anonymisierten Großkollektiven der industriellen Massengesellschaft.

Das moderne (Zeichen-)Geld erscheint innerhalb des für die Moderne konstitutiven dichotomen Deutungsrahmens von privater versus öffentlicher Sphäre als zentrales Medium 
solcher Vergesellschaftung und zugleich als Symbol der Ambivalenzen der Moderne: Versachlichung und Instrumentalisierung von Beziehungen einerseits, andererseits aber auch individuelle Freiheit(en) versprechende Freisetzung aus verbindlichen personalen Verpflichtungs- und Abhängigkeitsverhältnissen. Indem im geldvermittelten sozialen Austausch die wechselseitige Bezogenheit auf konkret-spezifische Andere von einer Wahlfreiheiten eröffnenden Abhängigkeit von Marktlagen abgelöst wird, weiten sich mit der Verfügbarkeit von Geld nicht nur die möglichen sozialen Kreise des Einzelnen. Darüber hinaus eröffnen sich für den Geldbesitzer auch expansive Teilhabe- und Aneignungschancen, allerdings verbunden mit der Kehrseite eines bedrohlichen existenziellen Exklusions- und Desintegrationsrisikos für Nichtgeldbesitzer. Wegen dieser individualisierenden Wirkung wird das moderne Geld im Kapitalismus, als >Substanz gewordene Wechselwirkung<, sowohl zum >fürchterlichsten Formzerstörer< wie zum >absoluten Mittel (Simmel 1989), welches in seiner >Vermögenseigenschaft< (Deutschmann 1999) jegliche Form von machtvoller gesellschaftlicher Teilhabe schlechthin verheißt. ${ }^{3}$ Weil diese >Macht des Geldes< (Deutschmann 2002) mit der in den zugrunde liegenden Tauschbeziehungen implizierten potentiellen Austauschbarkeit der Tauschpartner einhergeht, gerät Geld dem modernen Denken zum Gegenspieler der hochspezifischen, an der einzigartigen Individualität eines alter ego ausgerichteten >Liebe<.

Mit dem bürgerlichen Ehe- und Kleinfamilienmodell wurde die in dem Gegensatz von >Liebe < und >Geld< symbolisch codierte Widersprüchlichkeit der beiden modernen sozialen Integrationsmodi von öffentlicher, geldvermittelter Vergesellschaftung und privater, emotional-affektiver Vergemeinschaftung mit den aus ihnen folgenden Handlungsorientierungen und -logiken gleichsam institutionell eingehegt und gezähmt (vgl. auch Wimbauer 2003, S. 129 ff.): Begründet auf vorgeblich >natürlichen Geschlechtercharakteren< (Hausen 1976; Hausen/Wunder 1992) mit ihrer - der Dichotomie von privat/öffentlich folgenden - Trennung von weiblichen und männlichen Lebenssphären, ließ sich die moderne >Normalfamilie< als Familienhaushalt mit geschlechtsspezifischer Rollenverteilung konzipieren. Damit werden die Familienmitglieder ausschließlich durch das männliche Familieneinkommen (und nur im Ausnahmefall aufgrund ökonomischer Notwendigkeit ggf. ergänzt durch weiblichen Zuverdienst) vergesellschaftet (Born 1993). Und zugleich werden beide Partner, legitimiert durch das romantische Liebesideal, ${ }^{4}$ mittels eines komplementären Arrangements von (männlicher) >Arbeit für Geld<, welches ihnen beiden bzw. allen Familienmitgliedern zugute kommen muss, und (weiblicher) $>$ (Versorgungs-)Arbeit im Haushalt< vergemeinschaftet. ${ }^{5}$ Wem das Geld wie gehört, wozu es beiträgt und was es ermöglichen soll, ist innerhalb dieses institutionellen Regimes vorgegeben und bis in entsprechende sozialstaatliche Regelungen hinein abgesichert (Dingeldey 2000).

3) Zur Soziologie des Geldes vgl. grundlegend z.B. Marx (1929), Simmel (1989), Weber (1972, 1988), Parsons (1951, 1967), Luhmann (1984, 1988), Habermas (1981), für eine kritische Diskussion z.B. Deutschmann (1999, S. 36 ff.), siehe auch insgesamt Deutschmann (2000) sowie Ganßmann (1996, 2002).

4) Gerade diese Legitimationsfunktion führt aufgrund der dem Reziprozitätsverhältnis der intimen Paarbeziehung unterliegenden Gemeinsamkeitsannahme der >wechselseitigen Liebe< dazu, die Frage nach geschlechtsspezifischen Ungleichheiten und Machtdifferenzen auszublenden, denn »wer liebt, fragt nicht, ob er >gerecht $<$ behandelt wird, ob er eine Gegenliebe >schuldet<, ob die Beziehung >de-

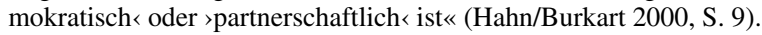

5) Damit ist idealtypisch jenes normative Modell der bürgerlichen Kleinfamilie skizziert, welches gemäß den gängigen familiendemographischen und haushaltsstatistischen Daten empirisch in Deutschland in den 1950er und 1960er Jahren - dem sogenannten >golden age of marriage < (vgl. Sieder 1987, S. 243 ff.) - seine weitgehende Gültigkeit und Verbreitung gefunden hat. Selbstverständlich lassen sich auch für jene Zeit z.B. mit Blick auf Erwerbstätigenziffern von Frauen (und damit wahrscheinlich auch im alltäglichen Umgang mit Geld) Abweichungen von diesem Leitbild finden, doch kann deren empirische Bandbreite vermutlich eher als gering eingeschätzt werden. Gleichzeitig dürften sie - bezogen auf den damals herrschenden und bis in die heutige Zeit reichenden Geschlechter- und Privatheitsdiskurs - wohl eindeutig als mehr oder weniger >abweichend « wahrgenommen worden sein. 


\subsection{Die Modernisierung der intimen Paarbeziehung: Von den (unterstellten) Individuali- sierungswirkungen des >eigenen Geldes auf >die Liebe}

Das male-breadwinner/female-housekeeper-Modell, welches durch die materielle Abhängigkeit der Frau wie durch die Verpflichtung des männlichen Einkommens als Familienversorgergeld die Geschlechter-Ungleichheiten innerhalb der intimen Paarbeziehung zementierte, unterliegt - gemäß dem familiensoziologischen Diskurs - derzeit einem grundlegenden Wandel: In einer wachsenden >Kultur der Kontingenz< des Privaten (Kaufmann 1995; Maihofer/Böhnisch/Wolf 2001) findet dieses Rollenmodell offenbar nur mehr eingeschränkte Zustimmung (z.B. Statistisches Bundesamt 2002, S. 533 ff.). Frauen streben nun nach selbstverdientem, >eigenem Geld<, welches, konnotiert mit eigenständigen beruflichen Sinnsetzungen wie Karriere, persönliche Entwicklung etc., nicht mehr lediglich Zuverdienst darstellt, sondern als >individuelles Geld< vor allem auch ein >eigenes Leben< ermöglichen soll (BeckGernsheim 1983). Die vor diesem Hintergrund formulierten Erwartungen für die Zukunft der Paarbeziehung und damit der Geschlechterverhältnisse lassen sich idealtypisch in folgenden zwei Entwicklungsszenarien darstellen, die beide hinsichtlich des seigenen Geldes< von Frauen und der damit angenommenen wachsenden Individualisierung letztlich abnehmende Ungleichheiten zwischen Männern und Frauen behaupten, aber mit für die Paarbeziehung bzw. für die Institutionen Ehe und Familie tendenziell gegenläufigen Folgen:

Im ersten Szenario wandelt sich die Rollenkomplementarität des alten Familienmodells grundlegend hin zu einer wachsenden Symmetrie zwischen den Lebenspartnern. Erst wenn beide Partner je individuell über eigene (finanzielle) Mittel (als Machtressourcen) verfügen, die sie, als wechselseitig gleichwertig anerkannt, in die gemeinsame Beziehung einbringen, sind gemeinsame Entscheidungen in der alltäglichen Beziehungsgestaltung im Sinne gleichberechtigter Partnerschaft in der Paarbeziehung überhaupt erst möglich. Durch einen >neuen< Umgang mit Geld, in dem das von beiden gleichermaßen in den Paar- bzw. Familienhaushalt eingebrachte Geld als gemeinsames, also gemeinsamer Verfügungsmacht unterliegendes wahrgenommen wird, werden Normen gleichberechtigter Partnerschaft, gleichen Einflusses und gleich verteilter Verfügungs- und Entscheidungsmacht in den alltagspraktisch bedeutsamen Lebensbereichen von Paaren verankert (z.B. Giddens 1992). Die damit verbundene, emanzipatorisch-vergemeinschaftend angelegte Argumentationsfigur lautet: Das individuell eingebrachte und gerade in dieser individuellen Zurechnung der Gemeinsamkeit dienende Geld befördert die Liebesbeziehung, welche nun, jenseits einseitiger materieller Abhängigkeiten und befreit von Ungleichheiten, deren Wurzeln im (ökonomischen) Außen der Beziehung liegen, die Authentizität affektueller Zuwendung verbürgt und so die Gestalt einer >demokratischen< Partnerschaft zweier gleichberechtigter Individuen annehmen kann.

Dem entgegen steht - im zweiten Szenario - die Befürchtung einer wachsenden Dominanz >individuellen Geldes<, welches in letzter Konsequenz das Fundament der Paarbeziehung als Liebesbeziehung infolge des »Förderns von Unabhängigkeit« der beiden Partner gleichsam von innen her zersetzen, auflösen, sprengen würde (z.B. Eggen 1994). Geld, selbst erworben, für einen selbst verfügbar und damit als >eigenes Geld< das >eigene Leben< innerhalb wie außerhalb der Beziehung ermöglichend und symbolisierend, unterliegt hier auch im Kontext der Paarbeziehung dem rationalen Kalkül individueller Nutzenmaximierung. Damit stünde innerhalb der Paarbeziehung das solcherart - weil nicht mehr durch institutionell legitimierte komplementäre Reziprozitätsnormen abgesicherte - >individualisierte< Geld der Partner in einem prekären Gegensatz zur Liebe (d.h. zur gesellschaftlich vorherrschenden Beziehungslegitimation). Der strukturelle Gegensatz von Geld und Liebe müsste dann von beiden Partnern in den alltäglichen Konstruktions- und Rekonstruktionsprozessen ihrer Paarbeziehung einschließlich der damit einhergehenden Wahloptionen und Entscheidungsprozesse mehr oder weniger reflexiv balanciert werden. Die Argumentationsfigur, nach der >(individuelles) 
Geld< (hier vor allem von Frauen) in letzter Konsequenz die >gemeinsame Liebe< be- bzw. verhindert, lautet: Soweit innerhalb der Paarbeziehung mit individuellem Geld die Gegensätzlichkeit von Geld und Liebe Platz greift, führt dies entweder zu dem letztlich aussichtslosen Unterfangen, diese interaktiv auflösen zu wollen, oder es erfolgt von vornherein ein Absprung aus der Beziehung, sofern die individuelle Kosten-Nutzen-Analyse negativ ausfällt. So oder so greift dieser Argumentation zufolge die rationale Marktlogik der Geldbeziehung auf die Paarbeziehung und die sie begründende und auf Dauer stellende Liebeslogik über.

\subsection{Ist Geld immer gleich Geld? - >Einfach-moderne` Grundannahmen zum Verhältnis von Liebe und Geld}

Beide Szenarien lassen sich als Beschreibungen eines grundlegenden Umbaus der (bürgerlich-modernen) Paarbeziehung als Basisinstitution der >einfachen Moderne< lesen. Doch sie folgen in ihren Grundannahmen und Argumentationsfiguren letztlich gerade jener modernen Liebes- und Geldsemantik, deren Wandel sie diskutieren, und verwischen so die Konturen der neuen Qualität dieses Wandels. Beide Varianten bedienen sich des Selbstbeschreibungsmodus der einfachen Moderne von der Gegensätzlichkeit geldvermittelter Vergesellschaftung versus affektiv-emotionaler Vergemeinschaftung und extrapolieren auf dieser Basis >linear< die Folgen >eigenen Geldes< (von Frauen) für die intime Paarbeziehung und das private Geschlechterverhältnis: Das erste, beziehungsoptimistische Szenario behauptet letztlich eine Purifizierung der >Liebe< durch die emanzipatorische Macht des Geldes. Das >eigene Geld< löst die in den überkommenen Beziehungs- und Geschlechterkonstellationen angelegte Verknüpfung von Geld und Liebe im Privaten, bereinigt die damit einhergehende Konfusion von Zuwendung und Versorgung, von existenzieller materieller Abhängigkeit und Autonomieverzicht. Noch deutlicher: Erst wenn sich die Lebenspartner auf einer materiell symmetrisierten Grundlage begegnen, die von jener wechselseitigen Gleichheit bestimmt wird, mit welcher sich idealtypisch Tauschpartner im öffentlichen Feld der Ökonomie gegenübertreten, kann emotional-affektive Vergemeinschaftung befreit und bereinigt von existenziellen materiellen Notwendigkeiten und Zwängen erfolgen. Überschätzt diese Deutung womöglich die Macht des Geldes im Privaten, sein emanzipatorisches Potential, so setzt das zweite Szenario kulturpessimistisch an jener >diabolischen < (Luhmann 1988) Potenz des Geldes an, welche - so die dort präsentierte Deutung - darin besteht, als nichtintendierte Nebenfolge seiner freisetzenden und individuell autonomisierenden Qualitäten die Exklusion des Sozialen, Gemeinschaftsbildenden zu betreiben. Mit der Beseitigung beziehungsimmanenter Machtverhältnisse und -asymmetrien wird so gleichsam die Beziehung selbst abgeschafft.

Unhinterfragt bleibt jedoch hier wie dort, ob Geld und Liebe >tatsächlich < (also in den heutigen Paarwirklichkeiten) je eigenen, gegensätzlichen Herstellungs- und (Aus-)Tauschlogiken unterliegen. Ungebrochen stehen sich in beiden Szenarien Geld und Liebe gegenüber: Hier die (romantische) Liebe, die höchst >subjektiv < genau zwei (bestimmte) Individuen miteinander vergemeinschaftet, diese aber je als ganze Person, womit deren Individualität gleichermaßen sowohl die Voraussetzung für ihre Beziehung darstellt als auch als deren Resultat erscheint; dort das in höchstem Maße >objektivierend « wirkende Geld, welches potenziell jede/n mit jedem/r in eine Beziehung treten lassen kann, im konkreten Austausch jedoch gerade von der Individualität der Beteiligten abstrahiert und sie ausschließlich als Träger der einzutauschenden Leistung anspricht und vergesellschaftet. Der Gegenpart von Liebe kann wiederum nur Liebe sein, Liebe ist in nichts anderes >konvertierbar<, während das im Geld enthaltene abstrakte Wertversprechen ausschließlich über den Tausch gegen etwas, das nicht Geld ist, einzulösen ist. Von dieser modernen >Liebes-Geld-Semantik gehen beide Szenarien aus, wenn sie unterstellen, dass Geld auch innerhalb der Paarbeziehung entsprechend der Marktlogik reiner Geldbeziehungen im Sinne eines >Mehr oder Weniger an >meinem/ 
deinem (unserem) Geld< funktioniert. So unterschiedlich dann die Folgen für die Stabilität von Paarbeziehungen ausbuchstabiert sein mögen, in beiden Fällen >wirkt< Geld als eigenes Geld immer >individualisierend $<$ auf die Paarbeziehung - in dem einen Szenario mit positiv, im anderen mit negativ bewerteten Folgen für die Paarbeziehung/Familie. Kurzum: Für (alle!) sozialen Beziehungen - und damit auch für die intime, am romantischen Liebescode ausgerichtete Paarbeziehung - scheint den skizzierten Argumentationen zufolge klar zu sein, was mit Geld (in seiner Gegensätzlichkeit zur Liebe) gemeint ist, d.h. was Geld >ist< und wie es >funktioniert<, wie es > wirkt<.

Was aber wäre, wenn jene (männlichen und weiblichen) Akteure, die >als Liebende $<$ ihre eigenen intimen Beziehungswelten konstruieren und auf Dauer zu stellen suchen, Geld - zumal dem >eigenen Geld< im Sinne selbst verdienten Einkommens - unterschiedliche symbolische Bedeutungen zuschreiben würden? Was wäre, wenn auch und gerade intime Paarbeziehungen heute nicht nur (wie schon immer) praktisch-materieller Sicherungen, sondern gerade auch ihrer je eigenen (geldvermittelten) symbolischen Ausdrucksweisen und Objektivationen bedürfen? Und was wäre, wenn je nach symbolischer Bedeutung für die Individuen-in-Paarbeziehungen und den damit korrespondierenden alltagspraktischen Mustern des Organisierens und Arrangierens von >eigenem und/oder gemeinsamen Geld< ganz unterschiedliche >individualisierende Wirkungsweisen<, Machteffekte und Ungleichheitsfolgen beobachtbar wären? Wären dies nicht Indizien für einen tiefgreifenden, möglicherweise grundlegenden und kategorialen Wandel der modernen Ordnung von Privatheit, für einen Umbruch moderner Basisinstitutionen und Basisprinzipien?

\section{Zum Zusammenhang von Geldarrangements, Beziehungskonzepten und Individualisierung: Empirische Befunde und theoretische Folgerungen}

Die empirischen Analysen zur individualisierenden Wirkung >eigenen< Geldes bei Doppelverdienerpaaren, die im Rahmen des SFB-Teilprojekts B6 durchgeführt wurden, ${ }^{6}$ zeigen, dass die Annahme, >eigenes< Geld würde sich als solches mehr oder weniger unmittelbar in >eigenes Leben und folglich in eine grundlegende Re-Konfiguration intimer Paarbeziehungen übersetzen, nicht haltbar ist. Geld als Medium von Vergesellschaftung hält keineswegs ungebrochen Einzug in die gemeinschaftliche Lebenswelt von Paaren, um dort, auf der Ebene des Privaten, eine ähnlich individualisierend wirkende Macht zu entfalten, wie dies Simmel (1989) für die modernen Wirtschafts- und öffentlichen Sozialbeziehungen gezeigt hat. So gibt es innerhalb von Paarbeziehungen keinen Automatismus, nach dem Geld etwa aufgrund der bloßen Quantitäten, in denen die Individuen-in-Paarbeziehungen z.B. aufgrund von Einkommensdifferenzen Zugriff auf Geld haben, wirkt. Vielmehr zeigt sich, dass Geld erst durch interaktive Bedeutungsarbeit der beiden Lebenspartner im Beziehungsalltag >wirklich< (gemacht) wird. Handlungsoptionen und -grenzen innerhalb oder außerhalb der Beziehung und die damit verbundenen Macht- oder Ungleichheitsrelationen in Paaren sind

6) Das Teilprojekt erforschte bislang ländervergleichend den Lebensalltag von Doppelverdienerpaaren und deren Geldarrangements anhand einer nichtstandardisierten Befragung von mehr als 40 Paaren in Deutschland, USA, Schweden und Spanien. Die für das deutsche Sample durchgeführten narrativen Paarinterviews (ergänzt um Individualinterviews mit wenigen Wochen zeitlichem Abstand) strukturierten sich anhand eines weitgehend offen gehaltenen Leitfadens, der neben den Geldarrangements verschiedenste Facetten des Beziehungsalltags der Paare thematisierte (z.B. Beziehungsbiographie, Erwerbstätigkeit, Hausarbeit). In einem zeitlichen Abstand von knapp drei Jahren erfolgte eine zweite Paarbefragung zu den Kontinuitäten oder Veränderungen im Paaralltag. Die erhobenen Interviewdaten wurden mit einer Kombination von Codierung und sequenzanalytischen Fallrekonstruktionen ausgewertet (vgl. Allmendinger/Schneider 2004; Wimbauer 2003; Schneider/ Wimbauer 2003; Wimbauer et al. 2002). An dieser Stelle danken wir allen ProjektmitarbeiterInnen, namentlich Ruth Christian, Holger Herma, Brigitte Rudolph und Tatjana Rosendorfer. 
demnach Resultat interaktiver Praktiken der Bedeutungszuschreibung von Geld und Liebe ( $>$ Liebe $<$ hier im Sinne von jeweils für die Partner geltenden Vorstellungen darüber, was ihre Paarbeziehung stiftet und auf Dauer stellt). Das heißt: Geld wird zum symbolisch wirksamen >Beziehungsgeld< (um-)gedeutet und entfaltet seine Individualisierungswirkungen und -effekte in den Lebenswelten von Intimbeziehungen in Abhängigkeit von den dort jeweils vorherrschenden Beziehungsvorstellungen und -bewertungen der Lebenspartner. Geld kann also ohne Frage innerhalb von Paarbeziehungen Ungleichheitswirkungen entfalten; dies geschieht aber nicht als Geldeffekt per se, sondern je unterschiedlich gemäß der Art und Weise, in der Geld symbolisch und alltagspraktisch in die Beziehungskonstruktionen der Paare eingebaut wird. Die folgenden Ausführungen zielen darauf, diese anhand exemplarischer Fallrekonstruktionen erarbeiteten empirischen Befunde (Allmendinger/Schneider 2004) insbesondere mit der Fokussierung auf ihre Individualisierungsfolgen - begrifflich-konzeptuell zu systematisieren und anschließend modernisierungstheoretisch zu verorten.

\subsection{Dimensionen von Geldarrangements in Paarbeziehungen}

Der erste zentrale empirische Befund lautet: Wenn von Geld in Paarbeziehungen die Rede ist, wenn seine Wirkungsweisen und Ungleichheitsfolgen analysiert werden sollen, reichen Kenntnisse über Höhe oder Verfügbarkeiten von Einkommen nicht aus (vgl. z.B. Blumstein/ Schwartz 1983; Pahl 1989; Vogler/Pahl 1993, 1994), sondern das Geldarrangement des Paares als solches muss in den Blick genommen werden. Mit >Geldarrangement $<$ bezeichnen wir nicht einfach z.B. das Vorhandensein gemeinsamer und/oder getrennter Konten, sondern der Begriff umfasst die Gesamtheit sowohl der symbolische(n) Bedeutung(en) von Geld aus der Sicht des Paares als auch die damit einhergehende alltagspraktische Organisation des Umgangs mit Geld (z.B. von der Kontenausstattung bis hin zu den jeweils vorhandenen Regelungen und symbolischen Praktiken des Paares, anhand derer die Geldpraxis im Alltag kontrolliert wird). Konkret sind hierbei drei Dimensionen auszuweisen:

\section{1) Mein - dein - unser Geld: Das >Relevant-machen von Geld im Paarkontext}

Mit dem je eigenen Einkommen beider Partner entsteht eine Situation, für welche die Beziehungsform der ersten Moderne keine institutionelle Antwort bereit hält, da nun die funktionale Erfordernis einer Verpflichtung des (ehemaligen Alleinverdiener-)Einkommens als Haushaltseinkommen entfällt. Die damit eintretende Deinstitutionalisierung des Geldes im Privaten macht es erforderlich, neue oder nunmehr neu zu erfindende >alte< Lösungen und Regelungen für die Behandlung des Geldes im Beziehungskontext und in die lebensweltliche Sphäre des Paares zu integrieren (Schneider/Wimbauer 2003). M.a.W. muss Geld erst gemeinschaftlich bedeutsam gemacht werden, was entlang folgender idealtypischer Muster des Zurechnens von mein - dein - unser Geld erfolgt, in denen sich die Individuen-im-Paar auch Art, Umfang und Grenzen ihrer wechselseitigen Verpflichtung und Vergemeinschaftung anzeigen.

Im ersten Typus - mit dem Grundprinzip der >gemeinsamen Kasse - erscheint jegliches Geld, auch wenn es aus einer Außenperspektive (z.B. juristisch) individuell zugeschrieben wird (etwa als Erwerbseinkommen), sofort durch die Paargemeinschaft kollektiviert und bleibt es auch aus der Perspektive der Lebenspartner, so dass jede Geldausgabe, auch wenn sie individuell getätigt wird, immer mit >gemeinsamem Geld< erfolgt. Im Gegenmodell der strikt >getrennten Kassen < findet sich eine individualistische Haushaltsführung mit durchgängig >eigenem Geld < der Partner. Hier erhält Geld seine Relevanz für die Beziehung gerade dadurch, dass es ausschließlich der individuellen Verfügbarkeit überantwortet bleibt und damit gleichsam außerhalb der gemeinsamen Beziehung gestellt ist. Zwischen diesen beiden (Extrem-)Mustern finden sich - als eigenständige Varianten - zwei weitere Möglichkeiten, bei denen in einem Fall das beim Eintritt in die Paarbeziehung kollektivierte Geld über entsprechende Bedeutungszuweisungen der Lebenspartner im Paaralltag (zum Teil) wieder in- 
dividuell zugeschrieben wird (z.B. als Taschengeld für jeden, das aus einer gemeinsamen Kasse entnommen wird, in die jegliche Einkünfte zunächst fließen); oder im anderen Fall, wenn das selbst verdiente Geld auch in der Paarbeziehung zunächst eigenes Geld bleibt (z.B. auf ein eigenes Konto geht), dann aber von den Partnern auch hier über entsprechende Bedeutungsarbeit in Teilen kollektiviert wird (indem damit gemeinsame Anschaffungen finanziert werden oder die Realisierung gemeinsamer Lebenspläne betrieben wird).

In dem relativ kleinen und hinsichtlich der je eigenen Erwerbstätigkeit von beiden Lebenspartnern gezielt homogen gewählten Paarsample fanden sich in verschiedenen realtypischen Ausprägungen und Mischformen alle vier Muster kollektivistischen/individualistischen $\mathrm{Zu}$ rechnens, die Paare einsetzen, um Geld überhaupt erst lebensweltlich in ihrer Paarbeziehung >wirklich< werden zu lassen. Deutlich wird: Inwiefern selbst verdientes Geld in die Paarbeziehung als eigenes Geld hineinwirkt, hängt offenbar von der Paarbeziehung bzw. den Geldund Beziehungsdefinitionen der Lebenspartner ab. Welches Geld wie, mit welcher Wertigkeit und nach welchen expliziten oder impliziten Regeln zugerechnet wird, kann jedoch nicht ohne Berücksichtigung zweier weiterer Dimensionen beantwortet werden.

\section{2) Geld ist nicht gleich Geld - zwei Bedeutungsformen von Geld}

Geld ist im Alltag von Paaren nicht gleich Geld, sondern wird von ihnen mit unterschiedlichen Wertigkeiten und Funktionen versehen, tritt gewissermaßen immer mehrfach codiert in Erscheinung (Nassehi 2005). Entlang der genannten unterschiedlichen Muster der Zurechnung von Geld als Beziehungsgeld produzieren die jeweiligen Geldarrangements nicht nur differente beziehungsinterne Anrechte auf das den Partnern verfügbare Geld - im Sinne lebensweltlicher >Besitztitel $<$ - sondern zugleich hinsichtlich ihrer beziehungskonformen Verwendbarkeit unterschiedliche Bedeutungsformen von Geld (vgl. auch Zelizer 1994). Rekonstruktiv-typisierend lassen sich hier zwei Formen unterscheiden: Reproduktions- oder Versorgungsgeld und Extensions- oder Distinktionsgeld (Hirseland/Schneider 2004).

Der Begriff >Reproduktions- bzw. Versorgungsgeld< legt zunächst die Vorstellung nahe, es diene der Abdeckung der absoluten (materiellen) Notwendigkeiten des alltäglichen Lebens und stünde somit jenseits variabler Zurechnungen und Bedeutungsverleihungen. Aus der Innenperspektive der im Projekt untersuchten Doppelverdiener-Paare bezeichnet der Begriff der >Notwendigkeit< jedoch mehr - nämlich alles, was das Paar zur Realisierung des von den Individuen-in-Paaren als >gemeinsam< definierten Ausschnitts der Beziehungswirklichkeit für erforderlich erachtet. Der Verwendungszweck dieses Geldes ist also auf die (materielle) Objektivation der miteinander geteilten Beziehungskultur bezogen - sozusagen durch die Festlegung einer gemeinschaftlichen, beziehungsspezifisch unterschiedlich umfangreichen Grundausstattung an Kollektivgütern, die damit das fraglose alltägliche >Unser< symbolisieren. Auch wenn keineswegs von vornherein ausgemacht ist, aus welcher (individuell oder kollektiv) zurechenbaren Quelle die erforderlichen Geldmittel stammen (müssen), so muss das beziehungs(kultur)spezifische Reproduktionsgeld doch regelmäßig von irgendwem und irgendwie aufgebracht werden. ${ }^{7}$ Von daher handelt es sich hier um (auf die Beziehung und die aus ihr resultierenden wechselseitigen >Verbindlichkeiten $<$ bezogenes) > verpflichtetes $<$ d.h. nicht mehr individuell frei disponibles Geld.

7) So deckt bei einem Paar die Frau mit ihrem Verdienst fast vollständig den Aufwand an >Reproduktionsgeld ; bei einem anderen Paar stellt die Frau ihre Eigentumswohnung inkl. Nebenkosten zur Verfügung, während der Mann von >seinem< Geld z.B. alltägliche Einkäufe übernimmt. Bei einem weiteren Paar hingegen existiert Reproduktionsgeld in diesem Sinne einer Verwendung für die Bereitstellung beziehungsinterner Kollektivgüter nicht, sondern alle finanziellen Aufwendungen der Partner sind strikt voneinander getrennt bzw. werden bei der alltäglichen Lebenshaltung entsprechend aufgeteilt und - das ist der entscheidende Punkt - adressieren somit eben nicht die gemeinsame Beziehung. 
In Abgrenzung dazu bezeichnet der Begriff des Extensions- bzw. Distinktionsgeldes jenes Geld, welches nach Abzug des Reproduktionsgeldes dem einzelnen Individuum-im-Paar zur freien Disposition verfügbar bleibt, d.h. innerhalb des durch das spezifische Geldarrangement gezogenen Rahmens >entpflichtet< ist und daher (marktvermittelte) Optionsräume auch jenseits der durch die Beziehung gesetzten Grenzen erschließt. Es eröffnet damit die Möglichkeit zur potenziellen Distinktion, Positionierung und Selbstentfaltung als Individuum jenseits des Paarzusammenhanges. Auch wenn es sich hier um individuell frei verfügbares, i.e.S. >eigenes $<$ Geld handelt, das im Gegensatz zum >gebundenen< Reproduktionsgeld das $>$ Mein< und das >Ich< der Individuen-im-Paar symbolisiert, muss der Sinnbezug seiner konkreten Verwendung dennoch nicht notwendig jenseits des jeweiligen alter ego, im >Außen der Beziehung liegen, sondern kann durchaus Element partnerbezogener strategischer oder taktischer Selbstdarstellung und Investitionen in die Partnerschaft sein (z.B. wenn die Frau nach einer Einkommensprämie ihren Mann durch eine Einladung zum Abendessen überrascht oder umgekehrt). Extensionsgeld bezeichnet also jenes Geld, durch welches sich das Individuum-im-Paar - je nachdem > gegen < oder >für < seinen Partner bzw. seine Partnerin, innerhalb oder außerhalb der Paarbeziehung - als >Selbst< ausdrückt und inszeniert, vom alltäglichen Lebensstil bis zu Freizeitaktivitäten etc.

Deutlich wird, dass die Produktion von Reproduktions- und Extensionsgeld ein Effekt der jeweiligen Geldarrangements ist. So kennt - idealtypisch formuliert - die >gemeinsame Kasse $<$ kein Extensions-/Distinktionsgeld, die >getrennte Kasse < hingegen kein Reproduktions-/ Versorgungsgeld.

\section{3) Geld und Nicht-Geld - Konvertibilität von Geld und Macht in Paarbeziehungen}

Die dritte relevante Dimension von Geldarrangements in Paarbeziehungen wird schließlich durch die Frage nach der $>$ Konvertibilität $<$ von Geld innerhalb der Beziehung umrissen (Hirseland 2004). Im Kontext der hier diskutierten Geldarrangements bezieht sich Konvertibilität auf die beziehungsinterne Einlösbarkeit von Geld gegen Nicht-Geld, z.B. gegen materielle Leistungen und/oder symbolisch wertvolle >Güter<, gegen Zeit, Liebe, Anerkennung etc. Wie in Abschnitt 2 diskutiert, gibt das bürgerliche Ehe- und Familienmodell als Basisinstitution der ersten Moderne auf die Frage nach dem symbolischen Tausch von Geld und Nicht-Geld zwischen den (als > sich liebend < geltenden) Lebenspartnern eine institutionell festgelegte Antwort. Im Gegensatz dazu zeigte die empirische Rekonstruktion der in den Interviews der von uns untersuchten Doppelverdienerpaaren dargestellten Alltagspraxis ihres jeweiligen (Beziehungs-)Geldarrangements, dass es für die Individuen-im-Paar ein symbolisch und institutionell offenes Feld gibt, in welchem subjektiv wahrnehmbare Gestaltungsspielräume implizite wie explizite Aushandlungen darüber ermöglichen, was in welchen Relationen überhaupt wie und gegen wessen (Reproduktions-/Extensions-)Geld einlösbar ist.

Mit der Konvertibilität sind somit nicht nur Fragen der praktischen Ausgestaltung des Beziehungsalltags verknüpft, sondern darüber hinaus liegt hier der Schlüssel zum Verständnis der Machteffekte von Geld innerhalb von Paarbeziehungen - soweit man Macht nicht im Weberschen Sinne lediglich als asymmetrisches Dominanzverhältnis aufgrund je individueller Merkmale konzipiert, sondern mit Foucault in einem relationalen Sinne auch als Ermöglichungsstruktur. Dann entscheidet die interaktiv hergestellte (Nicht-)Zulassung von Konvertibilität darüber, ob und inwieweit Geld im Binnenraum der Paarbeziehung >entmachtet< wird oder nicht, wie sich ungleich viel Geld (der Lebenspartner) in Abhängigkeit von den spezifischen Geldarrangements in ihren Machtbeziehungen auswirkt. So kann nach unseren Beobachtungen die im Arrangement der strikt getrennten Kassen institutionalisierte Nichtkonvertibilität von Geld nicht nur bedeuten, dass die Ausgestaltung des Beziehungsalltags an den finanziellen Möglichkeiten des geringer verdienenden Partners ausgerichtet wird, sondern dass sich auch die für den besser verdienenden Partner vergleichsweise höhere Ver- 
fügbarkeit von Extensionsgeld sich nicht in gemeinsame Aktivitäten - etwa einen gemeinschaftlichen kostenintensiven Urlaub - umsetzen lässt; ebenso wie das Zulassen von Konvertibilität unter Umständen zu einer eher traditional ausgerichteten Ausgestaltung von Partnerverhältnissen tendieren kann.

\subsection{Ko-/Kontra-Individualisierung als Effekt von Geldarrangements}

Das Verhältnis von Geld und Nicht-Geld muss also von den Akteuren im Beziehungsalltag als permanente interaktive Definitions- und Konstruktionsleistung bewältigt werden: $\mathrm{Ob}$ und welches Geld (Reproduktions- oder Extensionsgeld) innerhalb der Paarbeziehung mit etwas, was nicht Geld ist, verrechnet werden kann oder nicht, ob es in für den Einzelnen, für den Partner oder für die gemeinsame Beziehung bedeutsames $>$ Nicht-Geld $<$ (z.B. in $>$ Liebe $<$, in gemeinsame oder individuelle Zeit, in Anerkennung etc.) umgewandelt werden kann oder nicht, bestimmt den Spielraum paarinterner Gestaltungsmöglichkeiten ebenso mit, wie die Produktion und Verteilung von Reproduktions- und Extensionsgeld durch die jeweiligen Geldarrangements. Beides zusammen ist entscheidend für die dem Individuum-im-Paar innerhalb der Beziehung legitimerweise durch Geld zu erschließenden, individualisierungsrelevanten Optionsräume. Hierzu können mit Ko- und Kontra-Individualisierung zwei beziehungstypische Modi der Öffnung und Schließung individueller Handlungsspielräume und -optionen der Lebenspartner sowie damit verbundener Zurechnungsmodi von Individuierungsbegehren unterschieden werden. Die analytische Differenz von $>$ Ko/Kontra $<$ beruht zunächst darauf, dass die Paarvergemeinschaftung nicht nur als >Individualisierungsbremse< $\mathrm{zu}$ verstehen ist, sondern selbst (mehr oder weniger direkt) individualisierend wirken kann. $\mathrm{Zu}$ unterscheiden ist also, ob die Zurechnung der Öffnung oder Schließung individueller Handlungsspielräume und -optionen aus Sicht der Akteure individuell auf sich selbst oder auf den Partner bzw. die Beziehung (als Paarbeziehung bzw. als Familie) oder extern (jenseits der Beziehung) ausgerichtet ist. Ko-Individualisierung erschließt dann für das Individuum solche Handlungsspielräume und -optionen, die entweder dem jeweiligen Partner oder der gemeinsamen Paarbeziehung zugerechnet werden. Kontra-Individualisierung hingegen eröffnet Handlungsspielräume und -optionen, deren Zurechnung nicht auf den Partner oder nicht auf die Paarbeziehung gerichtet ist, also sich selbst oder extern zugerechnet werden, aber gegen den Partner oder gegen die Beziehung durchgesetzt werden müssen (Allmendinger/Schneider 2004; Allmendinger et al. 2004; Wohlrab-Sahr 1997).

\subsection{Beziehungskonzepte}

Mit dem je eigenen Einkommen beider Partner ist dem einfach-modernen Beziehungstypus nach dem Modell des bürgerlichen Ehe- und Familienideals eine Säule der fraglosen Geltung jener für die erste Moderne charakteristischen Ordnung von Geld und Liebe entzogen. Damit haben die sich in intimen Paarbeziehungen vergemeinschaftenden Individuen nicht nur die Chance zur Selbst-Gestaltung ihres Zusammenlebens, sondern müssen diese im Sinne eines nunmehr unhintergehbaren Wahlzwangs ergreifen und Wege zur Re-Institutionalisierung ihres Zusammen- oder Miteinanderlebens finden, deren Objektivation die eben skizzierte Kreation von >Beziehungsgeld < in und durch spezifische Geldarrangements bildet. Die skizzierte Dimensionierung von Geldarrangements in Paarbeziehungen stellt einen Zusammenhang her zwischen beobachtbarer Oberfläche der formalen Geldorganisation innerhalb der Beziehung und der Bedeutung, die diese für die Individuen-in-Paaren hat, und verweist so auf die expliziten und impliziten Vorstellungen der Individuen-in-Paaren darüber, was aus ihrer jeweiligen Perspektive konstitutiv für ihre eigene Paarbeziehung ist und diese auf Dauer stellt. D.h.: Wie sich Geldarrangements nun typischerweise ausgestalten, welche Zurechnungen und Geldformen vorherrschen und welche $>$ Währungen $<$ dabei bedeutsam sind, steht in direktem Zusammenhang zu den jeweiligen >Beziehungskonzepten $<$ der Paare (Schneider/Wimbauer 
2003; Wimbauer 2003; Wimbauer/Schneider/Ludwig-Mayerhofer 2002). Diese fungieren gleichsam als nicht unbedingt gewusste, vielmehr latent wirksame >Grammatiken < der Beziehung, die keineswegs immer von beiden Partnern geteilt werden. Und sie bestimmen als unhintergehbare normative Folien für Gestaltungsprinzipien des Alltags von Paaren den Aushandlungsspielraum ihrer jeweiligen Geldarrangements sowohl hinsichtlich der symbolischen Gehalte und Wertigkeiten wie auch der daraus resultierenden konkreten Zuweisungs-/ Verteilungs- sowie Kontrollpraktiken von Geld. Die im Fallmaterial rekonstruierten Beziehungskonzepte lassen sich zu zwei Idealtypen kondensieren, die mit unterschiedlichen Individualisierungspotentialen verknüpft sind (Hirseland/Schneider 2004; Allmendinger/ Schneider 2004).

Individualistische Beziehungskonzepte folgen der Maxime, dem Individuum-im-Paar größtmögliche Handlungsspielräume und Optionen offen zu halten. Sie richten sich nach dem Wertprinzip der Maximierung individueller Freiheit und beruhen auf einer Äquivalenzlogik des Austauschs im Sinne eines Vertragsmodells. Die Intimbeziehung wird so als ein prozessuales, modifizierbares und letztlich auflösbares Sozialverhältnis gedacht, dessen Bestand von situativen und okkasionellen Gegebenheiten abhängig ist und das stets neuer Entscheidungen und Vereinbarungen der Akteure bedarf. Dem entspricht die Orientierung der in einer Beziehung erforderlichen Austauschleistungen (des Gebens und Nehmens) am Äquivalenzprinzip der Marktökonomie; damit soll das wechselseitige Verpflichtungsprinzip von Schuld und Dankbarkeit aufgelöst werden, das in traditionalen Sozialbeziehungen dominiert. Trotz dieser >individualistischen Austauschlogik < kann in diesem Modell die Paarbeziehung durchaus als Erweiterung eigener Handlungsoptionen wahrgenommen werden oder solche überhaupt erst ermöglichen. Jedoch resultiert aus der unmittelbaren Reziprozität des Äquivalententausches als permanenter Ausgleich im Hier und Jetzt ein gesteigertes Spannungsfeld zwischen Ko- und Kontra-Individualisierung, denn der Freiheitszuwachs, der Freisetzungsgewinn des Einen darf keinesfalls auf Kosten des Anderen gehen (gleichgültig, ob innerhalb oder außerhalb der Beziehung). Individualistische Beziehungskonzepte scheinen dann zu >funktionieren< (im Sinne einer Verstetigung der Paarbeziehung), wenn sie von beiden Partnern geteilt werden, während Spannungen vor allem dann auftreten, wenn die Beziehungskonzepte der Individuen-in-Paaren nicht übereinstimmen (also ein individualistisches Beziehungskonzept nur von einem Partner verfolgt wird).

Kollektivistische Beziehungskonzepte weisen in die entgegengesetzte Richtung. Sie orientieren sich am Sozialgebilde >Paar als einer vergemeinschaftenden Institution sui generis und nehmen diese als Eigenwert wahr. Daraus folgt als handlungsleitendes Wertprinzip, dass der Bestand der Beziehung gegenüber der Abwägung individueller Vorteils- oder Chancenstrukturen Vorrang hat; diesem Prinzip zufolge >investiert< man - in der Selbstdeutung der Akteure - nicht in sich selbst oder den anderen, sondern in die gemeinsame Beziehung (insofern beruht auch dieser Typus auf einem modernen Verständnis von Individualität, ist also keinesfalls mit dem traditionalen Ehe-/Familienmodell gleichzusetzen). Für die sich auf diese Weise vergemeinschaftenden Individuen-in-Paaren steht der Aspekt der Dauerhaftigkeit von Beziehung sowohl als Bestandsgröße wie auch als alltäglich-selbstverständliche Rahmung der Wirklichkeit im Vordergrund.

Sind individualistische Konzepte auf die Beziehung als eine als flexibel definierte Größe bezogen, so erscheinen kollektivistische Konzepte insofern als fixiert, als solche Beziehungen (und die entsprechenden Relationen der Partner untereinander) von den Individuen-inPaaren als dauerhaft und nicht hinterfragbar wahrgenommen werden und so einen festen Rahmen bilden, der als Hintergrundfolie des je eigenen Handelns als gegeben in die Zukunft projiziert wird. Kollektivistische Beziehungskonzepte orientieren sich in ihren internen Funktionsbedingungen eher am Modell der Arbeitsteilung und beinhalten daher tendenziell differentielle Partnerkonzepte, die - wie z.B. in der bürgerlich-modernen Variante - komple- 
mentär an der Geschlechterachse ausgerichtet sind (vgl. Parsons 1951). Die Wertbasis solcher Beziehungskonzepte bildet (idealtypisch formuliert) die Solidarität zwischen den differentiell konzipierten Partnern und die Anerkennung inkommensurabler Eigenwerte. Differenz lässt sich so nicht (etwa im Sinne eines Mehr oder Weniger) vergleichen, sondern nur als gegebene Vielfalt und Pluralität anerkennen. Individualisierung tritt im Rahmen solcher Konzepte primär in Form von Ko-Individualisierungen in Erscheinung, denn KontraIndividualisierungen stellen die Legitimationsbasis der Solidarität und dadurch vermittelt des Beitrags zum Gemeinsamen im Sinne des (Fort-)Bestands des Modells in Frage. Zwar erzeugen derartige Konzepte Differenz, sind aber dennoch restriktiv hinsichtlich der Eröffnung von aus der Binnenperspektive wählbaren Handlungsoptionen. Im Gegensatz zu individualistischen weisen kollektivistische Beziehungskonzepte kein entlang symmetrisierend wirkender Egalitätsnormen objektivierbares Konzept von Gerechtigkeit auf. Als funktionales Äquivalent hierfür dient das Vertrauen darin, dass subjektiv empfundene Ungleichgewichte sich langfristig ausgleichen (aufgeschobene Reziprozität) oder belohnt würden.

Hinsichtlich des Zusammenhangs dieser beiden Typen von Beziehungskonzepten mit konstitutiven Merkmalen von Geldarrangements lässt sich zusammenfassend festhalten: Kollektivistische Beziehungskonzepte bilden die Grundlage für kollektive Zurechnungsmuster von Geld innerhalb der Paarbeziehung, entsprechendes gilt für den Zusammenhang von individualistischen Beziehungskonzepten und individuellen Zurechnungsmustern. Während kollektivistische Beziehungskonzepte dem Reproduktionsgeld einen hohen Stellenwert einräumen und Extensionsgeld lediglich vom Primat der Beziehung her ableiten und zulassen können (z.B. indem die Sinnreferenz von Extensionsgeld dann ko-individualisierend auf den/die Partner/in oder die Beziehung gerichtet ist und nicht kontra-individualisierend auf beziehungsexterne Orientierungen als >Selbst-Verwirklichung < jenseits der Beziehung), fokussieren individualistische Konzepte ko- und/oder kontra-individualisierendes Extensionsgeld unter möglichst weitgehender Negierung von Reproduktionsgeld. Für kollektivistische Beziehungskonzepte ist innerhalb der Beziehung eine Konvertibilität von Geld in nichtmonetäre (oder sonst wie durch Äquivalentenlogik bestimmbare) Werte oder Leistungen wie z.B. Anerkennung, Zuneigung im Kontext romantischer Liebe oder auch von Eltern-Liebe nicht nur möglich, sondern für das Funktionieren der Beziehung unabdingbar. Bei beiderseitigen individualistischen Beziehungskonzepten hingegen ist Konvertibilität von Geld (im idealtypischen Modellfall) innerhalb der Paarbeziehung ausgeschlossen.

\section{Macht und Ohnmacht des Geldes im Privaten - Die Modernisierung von Geldarrangements im Beziehungsalltag von Paaren}

\subsection{Geldarrangements und Individualisierungsdynamiken im paarbiographischen Zeit- verlauf}

Wie die jeweiligen Beziehungskonzepte Paare zu unterschiedlichen Anpassungen an geänderte Lebenssituationen zwingen und dabei gerade durch die damit einhergehenden Geldarrangements Individualisierungsoptionen und -grenzen formieren, soll anhand einer kurzen Gegenüberstellung von solchen Paaren mit individualistischen und kollektivistischen Beziehungskonzepten gezeigt werden, in deren Geldarrangements sich sowohl Reproduktions- als auch Extensionsgeld findet.

Gerade bei Doppelverdienerpaaren, wie den von uns untersuchten, verfolgen beide Partner eine eigenständige biographische Perspektive. Für das Paar geht damit die Anforderung einher, im Zeitverlauf der Beziehung insbesondere das Problem der biographischen Synchronisation lösen zu müssen (Hirseland/Herma/Schneider 2005). Nicht zuletzt aufgrund der beiderseitigen Berufsorientierung ergibt sich so ein - gegenüber dem bürgerlich-modernen 
Beziehungstypus der ersten Moderne - veränderter Interdependenzzusammenhang zwischen den Partnern, der aufgrund der für die zweite Moderne kennzeichnenden Abnahme planbarer und erwartbarer biographischer Normalverläufe (vgl. Bonß/Zinn in diesem Band) den Erwartungshorizont der gemeinsam geteilten wie individuell-persönlichen Zukunft dekonturiert und unter Ungewissheit stellt. Dabei sind unter den Auspizien der zweiten, reflexiven Moderne die Risiken (und Chancen) nicht mehr allein dem >Außen $<$ der Beziehung zuzurechnen, sondern auch den jeweiligen Beziehungskonstruktionen selbst, die aufgrund ihrer Beziehungskonzepte und Geldarrangements mit an der Konstruktion ihrer spezifischen Risikoumwelten beteiligt sind. D.h.: Welche Ereignisse (wie Arbeitslosigkeit, Elternschaft, aber auch Karrieresprünge und Geldgewinne) in welcher Form als Risiko gelten und im Falle ihres Eintretens wie und mit welchen Folgen für die Individuen-im-Paar und die Beziehung bewältigt werden können, wird mit entschieden durch die paarspezifischen Zurechnungen von >Mein-dein-unser $<$ und die in den jeweiligen Geldarrangements vorgezeichneten Bewältigungsstrategien entlang der ihnen zugrundeliegenden Beziehungskonzepte und ihrer Identitätsentwürfe für die Lebenspartner.

Entsprechend zeigte sich innerhalb des von uns untersuchten Samples, dass paarbiographische Veränderungen über die Zeitachse hinweg zwar durchaus zu schwachen Modifikationen der Geldarrangements - z.B. durch einnahmen- bzw. ausgabenorientierte Anpassung und Intensivierung von Kontrollregimes - führten, die Beziehungskonzepte der Paare bzw. Lebenspartner jedoch weitestgehend unverändert blieben. Komplexe Umbauten der Geldarrangements und grundlegende Umdefinitionen der Beziehungskonzepte werden vor allem bei jenen Paaren virulent, in denen die Lebenspartner (zum Zeitpunkt des Erstinterviews) divergierende Beziehungskonzepte verfolgten. ${ }^{8}$ Analysiert man die Konsequenzen der Geldarrangements im Zeitverlauf und vor dem Hintergrund der jeweiligen Beziehungskonzepte der Paare, so lässt sich gleichwohl zeigen, dass sich aus den paarspezifischen Geldund Beziehungskonstellationen typische Eigendynamiken ergeben, die die Individuen-inPaaren innerhalb ihrer Beziehung - zumal bei Eintritt von einschneidenden Lebensereignissen - unter je charakteristische Individualisierungszwänge setzen.

Wenn die Beziehung gemäß einer individualistischen Konzeption von beiden Partnern als Ort und Institution für die wechselseitige Förderung von Individualität und individuellen (beruflichen) Kompetenzen betrachtet wird, beruht in Entsprechung dazu - als Bedingung der Möglichkeit einer wechselseitigen Anerkennung der jeweiligen Individualität des anderen - das Geldarrangement auf einer strikten Separierung von Geld als je eigenes. Daher werden eintretende (paar-)biographisch relevante Ereignisse wie etwa Arbeitslosigkeit oder Schulden (des einen) selbst dann, wenn dies ökonomisch möglich wäre, nicht durch einen solidarischen paarinternen Ausgleich bewältigt (z.B. hätte in einem Fall ein unverhoffter Geldgewinn eines Partners als eigenes Extensionsgeld zum Schuldenabbau des anderen in Reproduktionsgeld umgewandelt werden können). Gemäß der vorherrschenden Beziehungsdefinition wird vielmehr - etwa bei Arbeitslosigkeit - die individuelle (wenngleich durchaus vom Partner unterstützte) Arbeit an der Verbesserung beruflicher Optionen zur Ausschöpfung sich bietender Chancenstrukturen optimiert wie ebenso das individuell-private Geldmanagement (um z.B. durch beruflichen Erfolg finanziell schnellstmöglichst wieder >voll auf eigenen Beinen<stehen zu können).

8) Die von zwei Paaren während des Untersuchungszeitraums vollzogenen Trennungen erscheinen als Folge von konfligierenden Beziehungskonzepten der Lebenspartner, die über den paarbiographischen Zeitverlauf hinweg keiner wechselseitigen Anpassung unterzogen wurden; vielmehr hat es den Anschein, als wären die (von 2 der 4 Akteure) neu eingegangenen Paarbeziehungen hinsichtlich der neu gewählten Partner eher eine weitere Annäherung an das ursprünglich schon in der ersten Paarbeziehung verfolgte Beziehungskonzept. 
Die Aufrechterhaltung des gemeinsam geteilten individualistischen Beziehungskonzepts verlangt in der paarbiographischen Entwicklung konsequent nach einer symmetrischen Lösung für die durch die Ereignisse im Leben des Partners (jedenfalls gemäß der Zurechnung durch die Beziehungskonstruktion) einseitig auftretenden Veränderungen. Sie erfordert letztlich also die gezielt und konsequent verfolgte Herbeiführung einer symmetrischen Lebenssituation für beide Partner (z.B. zwingt der bessere Job des einen den anderen zu weiteren Karriereanstrengungen; oder der Arbeitsplatzverlust erfordert den sofortigen Wiedereintritt in die Erwerbswelt, etwa durch den Schritt in die Selbständigkeit, dem dann womöglich sogar der andere Partner unter Aufgabe seines Arbeitsplatzes folgt). Individualistische Beziehungskonzepte forcieren somit eigendynamisch über den Zeitverlauf typischerweise eine Transformation der durch Veränderungen auf Seiten eines Partners eröffneten Optionen zur einseitigen Kontra-Individualisierung in Vorgaben für den anderen Partner zur unmittelbar nach- und mitzuvollziehenden Ko-Individualisierung. In der symbolischen Mikro-Ökonomie der Paare gäbe es ansonsten, ohne eine grundlegende Änderung des Beziehungskonzepts, infolge der fehlenden Konvertibilität von Geld nur die Alternative, entweder Gleichheit beim Reproduktionsgeld zu erzeugen und unterschiedliche Verfügbarkeiten individuellen Extensionsgeldes in Kauf zu nehmen, oder gleiche Verfügbarkeit von Extensionsgeld zu produzieren und ungleiche Beiträge zum Reproduktionsgeld zu tolerieren. Beide >Ungleichheiten sind auf Dauer aber kaum mit dem Beziehungskonzept zu vereinbaren. Die intendierte Individualisierung beider Partner qua Beziehungsdefinition - also die subjektive Seite von Freisetzungsprozessen als Individuierungsbegehren - schlägt damit paradoxerweise in ihr Gegenteil um, indem sie, als nichtintendierte Nebenfolge, in die Homogenisierung des je Individuellen mündet. Derart individualistische Beziehungskonzepte finden also dort ihre Grenzen von Individualisierung, wo sich zum einen - als Scheitern dieses Beziehungskonzepts - Differenzen und Asymmetrien zwischen den Lebenspartnern als wahrgenommene Ungleichheiten über den Zeitverlauf verstetigen und verfestigen. Und zum anderen produzieren sie in ihrem Erfolg der wechselseitigen Entpflichtung der Lebenspartner (fehlendes oder bedeutungsloses Reproduktionsgeld und nicht vorhandene Konvertibilität) bei gleichzeitiger maximaler Synchronisation der Ko-/Kontra-Individualisierungsprozesse als Egalitätsparadox ihre eigene Grenze von Individualität.

Bei kollektivistischen Beziehungskonzepten lässt sich eine gegensätzliche Entwicklungsdynamik bei gleichzeitig ähnlichen Individualisierungseffekten finden: Hier wird die Beziehung bzw. der Lebenspartner nicht als >Ressource < betrachtet, die es zu optimieren gilt, um so die Chancen eigener Selbstoptimierung zu erhöhen, sondern es gilt die Beziehung selbst durch individuelle Beiträge zu fördern. Entsprechend beruht das Geldarrangement auf dem Konzept der gemeinsamen Kasse, zu der jeder möglichst viel beizutragen trachtet, um dann aus dem Gemeinsamen schöpfen zu können. Eigenes Geld ist also zunächst per se gemeinsames Geld, aus dem die Lebenspartner das Reproduktions- und ihr jeweiliges Extensionsgeld entnehmen. Eintretende (paar-)biographisch relevante Ereignisse können hinsichtlich ihrer geldvermittelten Auswirkungen und darüber hinaus aufgrund der Verfügbarkeit über Reproduktionsgeld sowie infolge der umfassenden Konvertibilität von Beziehungsgeld scheinbar flexibler bewältigt werden. Auch in zeitlicher Hinsicht erlaubt dieses Beziehungskonzept mit seinem zeitlich gestreckten wechselseitigen Verpflichtungs- und Abhängigkeitsgefüge eine aufschiebende Abfederung bei der Bewältigung von Lebensereignissen ebenso wie bei längerfristigen einseitigen Investitionen (etwa in Bildung), die sich bei ihrer Einlösung dann für alle >auszahlen< (können). So mag z.B. die Arbeitslosigkeit des einen durch das gemeinsame Beschneiden von individuellen Extensionsgeldern überbrückt werden; nach dem gleichen Muster können individuelle (z.B. berufliche Fort-)Bildungsbestrebungen gemeinsam >subventioniert< werden, dienen sie doch letztlich im Rahmen dieses Konzepts dem Fortkommen der Beziehung als solcher und damit auch den Individuen in dieser Paarbeziehung. 
Auch wenn damit in der Vorherrschaft von Reproduktionsgeld jegliche Form von KontraIndividualisierung weitgehend ausgeschlossen oder zumindest minimiert bleibt und somit Modi der Ko-Individualisierung dominieren, folgt daraus eine Individualisierungsdynamik, die - nicht unähnlich zu individualistischen Konzepten - für die Lebenspartner dem Prinzip des >Alles oder Nichts < für alle oder niemandem entspricht. Obwohl auf Differenz basierend, können innerhalb dieses Beziehungskonzepts wahrgenommene Ungleichheiten zwischen den Lebenspartnern nur solange toleriert werden, als ihre Sinnreferenz an der gemeinsamen Beziehung orientiert ist. Insofern liegen die Grenzen von Individualisierung hier gerade darin, dass die über Beziehungsgeld vermittelte Vergemeinschaftung auch in diesem Beziehungskonzept die gleichgerichtete Individualisierung der Lebenspartner vorantreibt - und zwar basierend gleichsam auf der Individualisierung der (Paar-)Gemeinschaft.

Insgesamt betrachtet mögen moderne Doppelverdienerpaare an der Oberfläche ihrer alltagspraktischen Ausgestaltung von symbolisch offenen und institutionell entsicherten Beziehungsgeld-Verhältnissen eine Vielfalt an Gestaltungs- und Orientierungsmöglichkeiten für sich als Individuen-in-Paaren wahrnehmen. Bei genauerer Betrachtung erscheinen die Ausgestaltungen von Geldarrangements und damit verbundene Individualisierungseffekte jedoch bezüglich der Beziehungskonzepte deutlich pfadabhängig. Insbesondere die Verwirklichung kontra-individualisierender Zuschreibungs- und Handlungsmuster stößt an Grenzen dessen, was gemäß den jeweiligen Beziehungskonzepten innerhalb der Paarbeziehungen als unhintergehbarer Kernbestand an Gemeinsamkeiten erforderlich erscheint. Aber auch Ko-Individualisierungen unterliegen solchen Begrenzungen, wenn etwa die Vorgabe symmetrischer Homogenisierung beider Partner dazu führt, dass Ungleichgewichte und Ungleichheiten zwischen den Partnern im Falle ihrer Verstetigung nicht mehr aufgefangen werden können.

\subsection{Modernisierungstheoretische Folgerungen: Beziehungsgeld als Ungleichheitsgene- rator und die Transformation von Herrschaftsbezügen im Privaten}

Die bisher referierten Befunde und Überlegungen zeigen, dass Geld in intimen Paarbeziehungen nicht gleich Geld im Sinne der Beschreibungen der ersten Moderne ist und keineswegs per se gleichermaßen >linear< individualisierend wirkt. Geld im Sinne von Beziehungsgeld wird im Paaralltag zu einem Medium (gemacht), welches entlang expliziter und impliziter paarspezifischer Wirklichkeitsdefinitionen und Handlungsregeln einen spezifischen normativen Rahmen für den Paaralltag bildet. Hierin zeigen sich die Lebenspartner in ihrem Umgang mit Geld an, was Geld (für sie) ist, für was es steht, was es ermöglichen kann und was nicht, wer von beiden wie über Geld verfügen kann, wer nicht - und damit auch, wer typischerweise für den jeweils anderen wer ist und wie (mit welchen Optionen und Zwängen) mit Blick auf die gemeinsame Beziehung im Paaralltag zu handeln hat. Mit der wechselseitigen, geldvermittelten Selbst-Darstellung und -wahrnehmung der Lebenspartner (als Individuen-im-Paar im Innen der Paarbeziehung sowie gegenüber dem Außen) beruht der Machteffekt von Geld im Privaten auf dieser interaktiv geleisteten Bedeutungsarbeit, in der das lebensweltlich >bedeutete< Beziehungsgeld für die beiden Partner (in Anlehnung an Goffman 1980; Foucault 1993) zu einer flexiblen >Technik des Selbst<wird, die vom Bekenntniszwang zu den Schwächen des eigenen Geldcharakters bis hin zur Arbeit an der Geldpersönlichkeit des anderen reichen kann (Schneider/Wimbauer 2003; Wimbauer/ Schneider/Ludwig-Mayerhofer 2002). Somit ist es nicht >das Geld<, sondern sind es solche Definitionen und Regeln, die qua >Beziehungsgeld < und dessen symbolischen wie praktischen Mikropolitiken des Gebens und Nehmens für die Individuen in ihrer Paarbeziehung unterschiedliche Handlungsspielräume eröffnen oder schließen und deren unterschiedliche Zurechnungen (auf sich selbst, den Partner bzw. die gemeinsame Beziehung) erlauben oder verhindern. 
Entgegen der üblicherweise unterstellten (Markt-)Geldlogik, nach der Geld als >eigenes Geld < immer - und damit auch in Paarbeziehungen - einen Machtzuwachs bedeutet und frei und unabhängig macht, muss demzufolge die Frage nach Macht in intimen Paarbeziehungen relational auf die Paarbeziehung als Feld wahrgenommener Öffnungen und Schließungen von Handlungsoptionen bezogen werden. Insofern resultieren Macht- und Ungleichheitsrelationen zwischen Individuen-in-Paaren nicht lediglich aus der (Nicht-)Verfügbarkeit über individuell verdientes Geld. Individualität, Individualisierung (als Ko- und/oder Kontra-Individualisierung) wird vielmehr zu einem Machteffekt der jeweils vorherrschenden Konstellationen von Beziehungskonzepten und damit einhergehenden Geldarrangements. Ohne die Berücksichtigung dieser Konstellationen bleiben Antworten auf die Frage nach den geldvermittelten Individualisierungsprozessen im Privaten (sowohl in der optimistischen Variante einer Zunahme an Wahlfreiheiten und Egalisierungschancen wie in der pessimistischen als Destabilisierung der intimen Zweierbeziehung) empirisch unzureichend.

Im Kontext einer Theorie (und Empirie) reflexiver Modernisierung weisen diese Überlegungen zunächst in Richtung einer Hybridisierung des Privaten, welche die für die erste Moderne (von Weber über Parsons hin zu Luhmann) in Anspruch genommene funktionale und semantische Ausdifferenzierung von >Geld versus Liebe< zunehmend dekonturiert. Habermas hat - wenn auch nicht direkt auf Paarbeziehungen bzw. Familien bezogen - mit seiner $>$ Kolonialisierungsthese < der Lebenswelt durch systemische Rationalitäten bereits eine ähnliche Tendenz diagnostiziert und unter Pathologieverdacht gestellt (weil er von einer Dominanz systemischer Rationalitäten über lebensweltliche Sozialzusammenhänge ausging), wohingegen die Theorie reflexiver Moderne auch die gegenüber den traditionalen Paarbeziehungen gewonnenen Freiräume der Individuen als Individualisierungsgewinne in Anschlag bringen kann. Beiden Sichtweisen steht jedoch entgegen, dass die von uns gefundenen Paarmodelle in ihrer Struktur und Verlaufsdynamik eine Tendenz sowohl zu einer Sozialstruktur der Uneindeutigkeit - was die Geschlechterverhältnisse betrifft - als auch damit zusammenhängend zu einer Auflösung bzw. Reformulierung jener für die erste Moderne kennzeichnenden Basisprinzipen von Liebe und Geld und der sie tragenden Basisinstitutionen Ehe/Familie/Haushalt einerseits, rationaler Ökonomie andererseits aufweisen. Die eigensinnigen symbolischen Aufladungen von Geld als >Beziehungsgeld< und die damit verbundene Notwendigkeit beständiger Aushandlungen von Beziehungskonzepten und Geldarrangements verweist zwar auf eine Freisetzung aus den Sicherheiten der traditionalen Ordnung der privaten Paarbeziehungen. Doch damit werden keineswegs die - mit Geld als symbolisch generalisiertem Kommunikationsmedium verbundenen - Prinzipien der Vergesellschaftung zur Grundlage des Zusammenlebens der Individuen-in-Paaren. Vielmehr kann empirisch von der Ausbildung einer neuen, zwischen den überkommenen sozialwissenschaftlichen Polarisierungen von Gemeinschaft (〉Privatheit $<$ ) und Gesellschaft (〉Öffentlichkeit $<$ ) zu verortenden Mischform ausgegangen werden: An die Stelle der klassischen strikten Trennung von (sachlich-rechenhaftem und damit berechnendem) Geld und (romantischer und somit interesseloser) Liebe, tritt nunmehr ein notwendiger und unhintergehbarer Verweisungszusammenhang zwischen beiden. Pointiert gesagt: Die moderne Liebe bedarf des Geldes als eines symbolischen Mediums alltäglichen Austausches und identitätsstiftender, im Sinne einer Technik des Selbst zu praktizierender Grenzziehungen, um sich selbst gegenüber dem signifikanten Anderen und als Paar im intimen Innen und gegenüber dem Außen zu artikulieren.

Mit der Kreation von Beziehungsgeld als Technik des Selbst durch die Individuen-in-Paaren verbindet sich - verglichen mit der Herrschaftslogik der ersten Moderne - eine Neuformierung von Macht- und Herrschaftsverhältnissen in Paarbeziehungen. Und damit wandeln sich auch die Bedingungen der Produktion von Ungleichheit im Paarzusammenhang gegenüber dem klassischen Paarmodell grundlegend: Das traditionell bzw. einfach moderne Herrschaftsmodell beruhte noch auf Eindeutigkeiten von Kontroll- und Befehlskompetenzen, 
Regeln und Anweisungen und damit einhergehenden positionalen Über- und Unterordnungsverhältnissen (vgl. auch Beck/Lau in diesem Band), die sich im Bereich der privaten Paarbeziehungen in patriarchalen Geschlechterregimes manifestiert hat. D.h.: Dort waren die Pfade der Ungleichheitsentwicklung durch die normative Bindekraft geschlechtsspezifischer Rollenarrangements (Alleinverdiener/Hausfrau und Mutter) vorgegeben. Wie gezeigt, beruht die von den Individuen-in-Paaren geschaffene eigensinnige, subjektivierte Institution des Beziehungsgelds, objektiviert in den jeweiligen Geldarrangements der Paare, hingegen primär auf alltäglichen Aushandlungsprozessen der Lebenspartner gemäß den mit den jeweiligen Beziehungskonzepten gesetzten Interaktionslogiken. Die neuen Ungleichheiten hingegen resultieren somit als nicht intendierte Nebenfolgen aus der im Beziehungsgeld ausgemünzten Individualisierungsdynamik selbst, und sie sind gleichsam die zwingende Folge aus dem emanzipatorischen Postulat von Egalität in der Paarbeziehung und seiner Maßgabe der prinzipiellen Symmetrisierung der Individuen-in-Paaren.

Die in den Geldarrangements und ihren Kontrollregimes zum Ausdruck kommenden Machtrelationen stehen nun - gemäß der These von Geld als einer Technik des Selbst - keineswegs nur für eine einfache (rationale) Ökonomisierung der Beziehung, sondern orientieren sich (gemäß unseren empirischen Befunden) an entsprechenden Selbstoptimierungsvorgaben und -strategien einschließlich ihrer semantischen Kontextuierung etwa in Form von Managementmetaphern (z.B. als Arbeit an sich selbst, als wechselseitiges Coaching etc.; vgl. auch den Beitrag von Kratzer in diesem Band). Der Umgang mit Geld, Geldmanagement als solches - im öffentlichen Diskurs >populär-verwissenschaftlicht<, expertisiert, gleichsam erfolgsgarantiert erlernbar gemacht (vgl. z.B. die entsprechende Ratgeberliteratur) - wird so im Paarkontext einerseits immer weniger als Quelle persönlich zurechenbarer Macht und Abhängigkeiten gesehen; andererseits erscheint es, in diesem Sinne (scheinbar) depersonalisiert, umso deutlicher und unhinterfragbarer als Ausdruck von Individualität und Identität, als Spiegel des Selbst bzw. der Beziehung. Vielleicht gerade deshalb gerät die angestrebte Versachlichung des Beziehungsgeld-Managements mitunter zu ihrer nichtintendierten alltagsweltlichen Wiederverzauberung - etwa wenn, wie bei von uns untersuchten Paaren, selbst programmierte computergestützte Buchhaltungssysteme in der Darstellung der Paare sich gleichsam als magische Praktiken einer optimierten Geldkontrolle inszenieren und dabei (im Gegensatz etwa zum fremderzeugten Kontoauszug) als selbst gestaltete Objektivationen das Gefühl von selbstverantworteter Kontrolle vermitteln (sollen). Folgt man der für die Akteure in ihrem (Paar-)Alltag relevanten Sinnbasis, verdichtet sich in solchen empirischen Beispielen alltagspraktischer Geldarrangements gleichsam exemplarisch die behauptete Transformation von Herrschaft, nach der das optimierte Verwalten/Steuern/Managen als neue, >abstrakte< Leitsemantik modernisierter Herrschaftstechnologien die moralische Selbst-Formierung der Subjekte in ihren Intimbeziehungen orientiert. Plakativ ausgedrückt: Der patriarchale, personalisierte Herrschaftstypus, nach dem der als Familienernährer und Haushaltsvorstand positionierte Mann über das Medium Geld umfassende Macht- und Herrschaftsansprüche (gegenüber Frau und Kindern) im Privaten unmittelbar geltend machen konnte, mutiert zum - Männer wie Frauen (wie Kinder) ergreifenden - Typus des depersonalisierten, Sachzwänge prozedierenden Finanzverwaltens. Diese durchaus ambivalente Neuordnung der paarinternen Machtbeziehungen erweckt den Anschein der Abnahme personaler Machtfülle, nur um in der Form von Beziehungsgeld der Ökonomie nicht als rationale, sondern als - mit Marx gesprochen - >moralischste aller Wissenschaften< den Einzug in die solchermaßen modernisierte Sphäre des Privaten zu bereiten. 


\section{Literatur}

Allmendinger, Jutta/Ludwig-Mayerhofer, Wolfgang/Schneider, Werner/Wimbauer, Christine (2004): Eigenes Geld - Gemeinsames Leben. Zur Bedeutung von Geld in modernen Paarbeziehungen. In: Beck, Ulrich/Lau, Christoph (Hg.): Entgrenzung und Entscheidung: Was ist neu an der Theorie reflexiver Modernisierung? Frankfurt a.M.: Suhrkamp, S. 307-325.

Allmendinger, Jutta/Ludwig-Mayerhofer, Wolfgang/von Stebut, Janina/Wimbauer, Christine (2001): Gemeinsam leben, getrennt wirtschaften? Chancen und Grenzen der Individualisierung in Paarbeziehungen. In: Beck, Ulrich/Bonß, Wolfgang (Hrsg.): Die Modernisierung der Moderne. Frankfurt a.M.: Suhrkamp, S. 203-215.

Allmendinger, Jutta /Schneider, Werner (2004): Gemeinsam leben, getrennt wirtschaften - Grenzen der Individualisierung in Paarbeziehungen. Ergebnisbericht/Fortsetzungsantrag des Teilprojekts B6, SFB 536 >Reflexive Modernisierung ‘. München: o.V.

Beck, Ulrich (1986): Risikogesellschaft. Auf dem Weg in eine andere Moderne. Frankfurt a.M.: Suhrkamp.

Beck, Ulrich/Lau, Christoph (Hrsg.) (2004): Entgrenzung und Entscheidung. Was ist neu an der Theorie reflexiver Modernisierung? Frankfurt a.M.: Suhrkamp.

Becker, Gary S. (1981): A Treatise on the Family. Cambridge/Mass., London: Harvard University Press.

Beck-Gernsheim, Elisabeth (1983): Vom »Dasein für andere« zum Anspruch auf ein Stück »eigenes Leben«: Individualisierungsprozesse im weiblichen Lebenszusammenhang. In: Soziale Welt 34, S. 307-340.

Beck-Gernsheim, Elisabeth (1998): Was kommt nach der Familie? Einblicke in eine neue Lebensform. München: C.H. Beck.

Berger, Peter L./Kellner, Hansfried (1965): Die Ehe und die Konstruktion der Wirklichkeit. Eine Abhandlung zur Mikrosoziologie des Wissens. In: Soziale Welt 16, S. 220-235.

Blossfeld, Hans-Peter/Drobnič, Sonja (Hrsg.) (2001): Careers of Couples in Contemporary Society. From Male Breadwinner to Dual-Earner Families. Oxford: Oxford University Press.

Blumstein, Philip/Schwartz, Pepper (1983): American Couples. Money, Work, Sex. New York: William Morrow.

Born, Claudia (1993): Das Einkommen im ehepartnerlichen Aushandlungsprozess. Argumentationsfigur zwischen Innovation und Restauration. In: Born, Claudia/Krüger, Helga (Hrsg.): Erwerbsverläufe von Ehepartnern und die Modernisierung weiblicher Lebensläufe. Status Passages and the Life Course 5. Weinheim: Deutscher Studienverlag, S. 191-208.

Dackweiler, Regina M./Hornung, Ursula (Hrsg.) (2003): frauen - macht - geld. Münster: Westfälisches Dampfboot.

Delphy, Christine/Leonard, Diana (Hrsg.) (1992): Familiar Exploitation: A New Analysis of Marriage in Contemporary Western Societies. Cambridge: Polity Press.

Deutschmann, Christoph (1999): Die Verheißung des absoluten Reichtums. Zur religiösen Natur des Kapitalismus. Frankfurt/New York: Campus.

Deutschmann, Christoph (2000): Geld als »absolutes Mittel«. Zur Aktualität von Simmels Geldtheorie. In: Berliner Journal für Soziologie 10, S. 301-313.

Deutschmann, Christoph (Hrsg.) (2002): Die gesellschaftliche Macht des Geldes. Leviathan Sonderheft 21. Wiesbaden: Westdeutscher Verlag.

Dingeldey, Irene (Hrsg.) (2000): Erwerbstätigkeit und Familie in Steuer- und Sozialversicherungssystemen. Begünstigungen und Belastungen verschiedener familialer Erwerbsmuster im Ländervergleich. Opladen: Leske + Budrich.

Eggen, Bernd (1994): Familie der Gesellschaft. Kontinuität im Wandel. Ludwigsburg u.a.: Verlag Wissenschaft und Praxis.

Foucault, Michel (1993): Technologien des Selbst. Frankfurt a.M.: Fischer. 
Ganßmann, Heiner (1996): Geld und Arbeit. Wirtschaftssoziologische Grundlagen einer Theorie der modernen Gesellschaft. Frankfurt/New York: Campus.

Ganßmann, Heiner (2002): Das Geldspiel. In: Deutschmann, Christoph (Hrsg.): Die gesellschaftliche Macht des Geldes. Leviathan Sonderheft 21. Wiesbaden: Westdeutscher Verlag, S. 21-46.

Giddens, Anthony (1992): The Transformation of Intimacy. Sexuality, Live and Eroticism in Modern Societies. Cambridge: Polity Press.

Goffman, Erving (1980): Stigma. Über Techniken der Bewältigung beschädigter Identität. 4. Auflage, Frankfurt a.M.: Suhrkamp.

Habermas, Jürgen (1981): Theorie des kommunikativen Handels, Band I und II. Frankfurt a.M.: Suhrkamp.

Hahn, Kornelia/Burkart, Günter (Hrsg.) (1998): Liebe am Ende des 20. Jahrhunderts (Studien zur Soziologie intimer Beziehungen I). Opladen: Leske + Budrich.

Hahn, Kornelia/Burkart, Günter (Hrsg.) (2000): Grenzen und Grenzüberschreitungen der Liebe (Studien zur Soziologie intimer Beziehungen II). Opladen: Leske + Budrich.

Hausen, Karin (1976): Die Polarisierung der >Geschlechtscharaktere «. Eine Spiegelung der Dissoziation von Erwerbs- und Familienleben. In: Conze, Werner (Hrsg.): Sozialgeschichte der Familie in der Neuzeit Europas - Neue Forschungen. Stuttgart: Ernst Klett, S. 367-393.

Hausen, Karin/Wunder, Heike (Hrsg.) (1992): Frauengeschichte-Geschlechtergeschichte. Frankfurt a.M.: Campus.

Herrmann, Horst (2001): Liebesbeziehungen - Lebensentwürfe. Eine Soziologie der Partnerschaft. Münster: Telos.

Hirseland, Andreas/Schneider, Werner (2004): Geldarrangements in Paarbeziehungen: Kontinuitäten und Veränderungsdynamiken - Ergebnisse der 2. Phase. Arbeitspapier des Projekts B6 des SFB 536. München: unveröff. Manuskript.

Hirseland, Andreas (2004): Ergebnisse der internationalen Paarvergleichsstudie. Arbeitspapier B6 des SFB 536. München: unveröff. Manuskript.

Hirseland, Andreas/Herma, Holger/Schneider, Werner (2005): Geld und Karriere - biographische Synchronisation und Ungleichheit bei berufsorientierten Paaren. In: Solga, Heike/Wimbauer, Christine (Hrsg): Wenn zwei das gleiche tun - Ideal und Realität sozialer (Un)Gleichheit in Dual Career Couples. Opladen: Barbara Budrich (im Erscheinen), S. 163-186.

Hoffmeister, Dieter (2001): Mythos Familie. Zur soziologischen Theorie familialen Wandels. Opladen: Leske + Budrich.

Hohenester, Birgitta (2000): Dyadische Einheit. Zur sozialen Konstitution der ehelichen Beziehung. Konstanz: Universitätsverlag Konstanz.

Illouz, Eva (2003): Der Konsum der Romantik. Liebe und die kulturellen Widersprüche des Kapitalismus. Frankfurt a.M.: Campus.

Kaufmann, Franz-Xaver (1995): Zukunft der Familie im vereinten Deutschland. Gesellschaftliche und politische Bedingungen. München: C.H. Beck.

Lenz, Karl (2003): Soziologie der Zweierbeziehung. Eine Einführung. 2., akt. und erw. Aufl., Opladen: Westdeutscher Verlag.

Ludwig-Mayerhofer, Wolfgang (2004): Geldverwaltung und -verteilung im (Familien-)Haushalt. Expertise im Rahmen des 7. Familienberichts des Bundesministeriums für Familie, Senioren, Frauen und Jugend (BMFSFJ).

Luhmann, Niklas (1982): Liebe als Passion. Zur Codierung von Intimität. Frankfurt a.M.: Suhrkamp.

Luhmann, Niklas (1984): Soziale Systeme. Grundriß einer allgemeinen Theorie. Frankfurt a.M.: Suhrkamp.

Luhmann, Niklas (1988): Die Wirtschaft der Gesellschaft. Frankfurt a.M.: Suhrkamp.

Maihofer, Andrea/Böhnisch, Tomke/Wolf, Anne (2001): Wandel der Familie (Arbeitspapier 48 der Hans Böckler Stiftung, Reihe: Zukunft der Gesellschaft). Düsseldorf: o.V. 
Marx, Karl (1929): Das Kapital. Kritik der politischen Ökonomie (Band 1). Leipzig.

Mead, George Herbert (1973 [1934]): Geist, Identität und Gesellschaft. Frankfurt a.M.: Suhrkamp.

Millhofer, Petra (1973): Familie und Klasse. Ein Beitrag zu den politischen Konsequenzen familialer Sozialisation. Frankfurt a.M.: Athenäum.

Nassehi, Armin (2005): Geld oder Leben. Essay für die Soziologische Revue (im Erscheinen).

Pahl, Jan (1989): Money and Marriage. Basingstoke, London: MacMillan.

Parsons, Talcott (1951): The Social System. Glencoe: The Free Press.

Parsons, Talcott (1967): Sociological Theory and Modern Society. New York: The Free Press.

Paul, Axel T. (2004): Die Gesellschaft des Geldes. Entwurf einer monetären Theorie der Moderne. Wiesbaden: VS Verlag.

Rubery, Jill/Smith, Mark/Fagan, Colette (1999): Women's Employment in Europe: Trends and Prospects. London: Routledge.

Schneider, Werner/Wimbauer, Christine (2003): Entstaatlichung >von unten < - Ungleichheit in Paarbeziehungen: Zur Transformation der institutionellen Ordnung von Geld und Liebe im Privaten. In: Allmendinger, Jutta (Hg.): Entstaatlichung und soziale Sicherheit. Verhandlungen des 31. Kongresses der Deutschen Gesellschaft für Soziologie in Leipzig. Opladen: Leske + Budrich, S. 707-722.

Schneider, Werner/Wimbauer, Christine (2004): Das eigene Geld von Frauen - Zur symbolischen Bedeutung von Geld in Paarbeziehungen bzw. in der Familie. Expertise im Rahmen des 7. Familienberichts des Bundesministeriums für Familie, Senioren, Frauen und Jugend (BMFSFJ).

Segalen, Martine (1998): Die industrielle Revolution: Vom Proletarier zum Bürger. In: Burguière, André/ Klapisch-Zuber, Christiane /Segalen, Martine/Zonabend, Françoise (Hrsg.): Geschichte der Familie. Band 4: 20. Jahrhundert. Frankfurt a.M.: Campus, S.13-58.

Sieder, Reinhard (1987): Sozialgeschichte der Familie. Frankfurt a.M.: Suhrkamp.

Simmel, Georg (1989 [1900]): Philosophie des Geldes. Gesamtausgabe Band 6. Frankfurt a.M.: Suhrkamp.

Tyrell, Hartmann (1987): Romantische Liebe - Überlegungen zu ihrer »quantitativen Bestimmtheit«. In: Baecker, Dirk et al. (Hg.): Theorie als Passion. Niklas Luhmann zum 60. Geburtstag. Frankfurt a.M.: Suhrkamp, S. 570-599.

Vogler, Carolyn/Pahl, Jan (1993): Social and economic change and the organisation of money within marriage. In: Work, Employment and Society 7, S. 71-95.

Vogler, Carolyn/Pahl, Jan (1994): Money, Power and Inequality Within Marriage. In: The Sociological Review 42, S. 263-288.

Walby, Sylvia (1990): Theorizing Patriarchy. Oxford: Blackwell.

Weber, Max (1972 [1921]): Wirtschaft und Gesellschaft. Grundriss der verstehenden Soziologie. Tübingen: J.C.B. Mohr.

Weber, Max (1988 [1920; 1916]): Die Wirtschaftsethik der Weltreligionen. In: Ders.: Gesammelte Aufsätze zur Religionssoziologie. Tübingen: J.C.B. Mohr, S. 237-573 (UTB 1488).

Wimbauer, Christine (2003): Geld und Liebe. Zur symbolischen Bedeutung von Geld in Paarbeziehungen. Frankfurt/New York: Campus.

Wimbauer, Christine/Schneider, Werner/Ludwig-Mayerhofer, Wolfgang (2002): Prekäre Balancen. Liebe und Geld in Paarbeziehungen. In: Deutschmann, Christoph (Hrsg.): Die gesellschaftliche Macht des Geldes. Leviathan Sonderheft 21. Wiesbaden: Westdeutscher Verlag, S. 263-285.

Wohlrab-Sahr, Monika (1997): Individualisierung: Differenzierungsprozeß und Zurechnungsmodus. In: Beck, Ulrich/Sopp, Peter (Hrsg.): Individualisierung und Integration. Neue Konfliktlinien und neuer Integrationsmodus? Opladen: Leske + Budrich, S. 23-36. 
Zelizer, Viviana A. (1994): The Social Meaning of Money. Pin Money, Pay Cheques, Poor Relief, and Other Currencies. Princeton: Princeton University Press.

Prof. Dr. Werner Schneider Universität Augsburg, Phil.-Soz.wiss. Fakultät,

Universitätsstr. 10, 86135 Augsburg. werner.schneider@phil.uni-augsburg.de

Dr. Andreas Hirseland

LMU München, Institut für Soziologie, Konradstr. 6, 80801 München.

AHirseland@aol.com

Prof. Dr. Wolfgang-Ludwig Mayerhofer Universität Siegen, FB 1, Adolf-Reichwein-Str. 2, 57076 Siegen. ludwig-mayerhofer@soziologie.uni-siegen.de

Prof. Jutta Allmendinger, Ph.D. Institut für Arbeitsmarkt und Berufsforschung (IAB),

Regensburgerstr. 104, 90478 Nürnberg. jutta.allmendinger@iab.de 